\title{
Exponential Decay of Wavelength in a Dissipative System
}

\author{
Li Hua Yu \\ National Synchrotron Light Source, Brookhaven National Laboratory, N.Y.11973
}

\begin{abstract}
Applying a technique developed in a recent work[1] to calculate wavefunction evolution in a dissipative system with Ohmic friction, we show that the wavelength of the wavefunction decays exponentially, while the Brownian motion width gradually increases. In an interference experiment, when these two parameters become equal, the Brownian motion erases the fringes, the system thus approaches classical limit. We show that the wavelength decay is an observable phenomenon.
\end{abstract}


1. Introduction. The simplest example of a dissipative system, an harmonic oscillator coupled to an environment of a bath of harmonic oscillators, has been the subject of extensive studies [2-4] (see [1] for more references). In a recent paper[1], we obtained a simple and exact solution for the wave function of the system plus the bath, in the Ohmic case. It is described by the direct product in two independent Hilbert spaces. One of them is described by an effective Hamiltonian, the other represents the effect of the bath, i.e., the Brownian motion, thus clarifying the meaning of the wave function of the effective Hamiltonian after the bath variables are eliminated, establishing a connection between two different approaches to the dissipative system problems: the effective Hamiltonian approach, and the system plus bath approach.

In this paper we shall apply this result to a specific case to show how the wavelength of the wavefunction decays exponentially while the Brownian motion width increases at the same time. We shall show that in an experiment with two overlapping wave packets interfering with each other, the interference term of the probability density decreases rapidly when the wavelength of the wavefunction of the effective Hamiltonian reduces to be equal to the Brownian motion width. Therefore, our result provides a transparent picture about how a quantum system, when interacts with environment and hence becomes a dissipative system, gradually evolves into a classical system.

First, we derive a simple formula for the probability density. Next, we apply our method to the case of an initial gaussian wave packet displaced from the center of the parabolic potential well, and show that the wavelength of the effective Hamiltonian decreases exponentially while the Brownian motion width increases. Then, we use the results to calculate the interference term, assuming the initial state is a superposition of two wave packets with minimum uncertainty, one of them is centered at the origin, the other is displaced from the center. This problem has been studied by A.O.Caldeira and A.J.Leggett (CL)[4]. However, the result does 
not show the expected fringe wavelength decay. This puzzle stimulated our further analysis given in the last section, which explains why the wavelength decay is not observable in this well studied case, and shows that if the initial wave packet width is different from that with the minimum uncertainty, the wavelength decay becomes an observable phenomenon. We shall use the same notation as the previous paper, and quote formulas from there.

2. The probability density. We consider an harmonic oscillator of frequency $\omega_{0}$, linearly coupled to a bath of harmonic oscillators of frequency $\omega_{j}$ with Ohmic spectral density distribution. The Hamiltonian of this system is given in reference [1]:

$$
H=\frac{p^{2}}{2 M}+\frac{1}{2} M\left(\omega_{0}^{2}+\Delta \omega^{2}\right) q^{2}+q \sum_{j} c_{j} x_{j}+\sum_{j}\left(\frac{p_{j}^{2}}{2 m_{j}}+\frac{1}{2} m_{j} \omega_{j}^{2} x_{j}^{2}\right) .
$$

It is well known that the coupling to the bath introduces damping to the harmonic oscillator [1]. The damping rate is $\eta$ and the system frequency is shifted to $\omega=\left(\omega_{0}^{2}-\eta^{2} / 4\right)^{1 / 2}$ by the damping. In the previous paper [1] we showed that the wavefunction of the system plus the bath, in the Schoedinger representation, at time t, can be written as:

$$
\Psi\left(q,\left\{\xi_{j}\right\}, t\right)=\psi\left(q-\sum_{j} \xi_{j}, t\right) \prod_{j=1}^{N} \chi_{j}\left(\xi_{j}, t\right)
$$

where the wavefunction $\psi(Q, t)$ is a solution of the effective Hamiltonian eq.(23) of [1]:

$$
H_{Q}=e^{-\eta t} \frac{P^{2}}{2 M}+\frac{1}{2} M \omega_{0}^{2} e^{\eta t} Q^{2}
$$

while $\xi_{j}=b_{j 1}(t) x_{j 0}+b_{j 2}(t) \dot{x}_{j 0}$ is the contribution of the $\mathrm{j}$ 'th bath oscillator to the Brownian motion with $x_{j 0}, \dot{x}_{j 0}$ its initial position and velocity. The coefficients $b_{j 1}(t)$ and $b_{j 2}(t)$ are well known in elementary physics[1]. The function $\chi_{j}\left(\xi_{j}, t\right)$ is given by $\chi_{j}\left(\xi_{j}, t\right)=<\theta_{\xi_{j}} \mid \chi_{j 0}>$, where $\chi_{j 0}\left(x_{j 0}\right)$ is the initial state of the bath oscillator, and $\mid \theta_{\xi_{j}}>$ is an eigenstate of the operator $\xi_{j}$ with eigenvalue $\xi_{j}$, determined to within an arbitrary phase, which depends on only $\xi_{j}$ : 


$$
\theta_{\xi_{j}}\left(x_{j 0}, t\right)=\left(\frac{m_{j}}{2 \pi \hbar b_{j 2}}\right)^{\frac{1}{2}} \exp \left[-\frac{i m_{j}}{2 \hbar b_{j 2}}\left(b_{j 1} x_{j 0}^{2}-2 x_{j 0} \xi_{j}\right)\right] .
$$

We are interested in the probability density time evolution if initially the bath is in an equilibrium at temperature T. Assuming initially the $j$ 'th bath oscillator is in an excited state $n_{j}$, we write the initial wavefunction of the this oscillator as $\chi_{j}\left(x_{j 0}\right)=\chi_{j}^{\left(n_{j}\right)}\left(x_{j 0}\right)$. Then, the probability density for finding the system at position $q$ and finding the contribution of the bath oscillator $\mathrm{j}$ to the Brownian motion to be $\xi_{j}$, is:

$$
\rho\left(q,\left\{\xi_{j}\right\} ; t\right)=\left|\psi\left(q-\sum_{j} \xi_{j}, t\right)\right|^{2} \prod_{j=1}^{N} \rho_{j}\left(\xi_{j} ; t\right),
$$

with the distribution of $\xi_{j}$ at time t:

$$
\rho_{j}\left(\xi_{j} ; t\right) \equiv\left(\sum_{n_{j}=0}^{\infty}\left|\chi_{j}^{\left(n_{j}\right)}\left(\xi_{j}, t\right)\right|^{2} e^{-\beta\left(n_{j}+\frac{1}{2}\right) \omega_{j} \hbar}\right) / Z_{j}
$$

where $\beta=1 / k T$, and the partition function is

$$
Z_{j}=\sum_{n_{j}=0}^{\infty} e^{-\beta\left(n_{j}+\frac{1}{2}\right) \omega_{j} \hbar}=\frac{1}{2 \sinh \left(\frac{\beta \hbar \omega_{j}}{2}\right)} .
$$

Using $\chi_{j}\left(\xi_{j}, t\right)=<\theta_{\xi_{j}} \mid \chi_{j 0}>$, we find $\rho_{j}\left(\xi_{j} ; t\right)=<\theta_{\xi_{j}}\left|\rho_{j 0}\right| \theta_{\xi_{j}}>$, where $\rho_{j 0}$ is the initial density matrix of the $\mathrm{j}$ 'th bath oscillator. The matrix elements of $\rho_{j 0}$ is well known in quantum statistics text book[3]:

$\rho_{j 0}\left(x_{j 0}, x_{j 0}^{\prime}\right)=\left(\frac{m_{j} \omega_{j} \tanh \left(\frac{\beta \hbar \omega_{j}}{2}\right)}{\hbar \pi}\right)^{\frac{1}{2}} \operatorname{Exp}\left\{-\frac{m_{j} \omega_{j}}{\hbar}\left[\frac{1}{2} \operatorname{coth}\left(\beta \hbar \omega_{j}\right)\left(x_{j 0}^{2}+x_{j 0}^{\prime 2}\right)-\frac{x_{j 0} x_{j 0}^{\prime}}{\sinh \left(\beta \hbar \omega_{j}\right)}\right]\right\}$. 
Hence, using eq.(4), followed by a straight forward calculation, we obtain (obviously the arbitrary phase of $\theta_{\xi_{j}}$ would not influence the result):

$$
\rho_{j}\left(\xi_{j} ; t\right)=\frac{1}{\sqrt{2 \pi} \sigma_{j}} e^{-\frac{\xi_{j}^{2}}{2 \sigma_{j}^{2}}}
$$

which is a gaussian distribution with width:

$$
\sigma_{j}^{2}=\frac{\hbar}{2 m_{j} \omega_{j}}\left(\left|b_{j 1}(t)\right|^{2}+\omega_{j}^{2}\left|b_{j 2}(t)\right|^{2}\right) \operatorname{coth}\left(\frac{\hbar \omega_{j}}{2 k T}\right) .
$$

The density matrix traced over the variables $\xi_{j}$ is given by:

$$
\rho\left(q_{1}, q_{2} ; t\right)=\underbrace{\iiint \ldots \int}_{N} \psi^{*}\left(q_{1}-\sum_{j} \xi_{j}, t\right) \psi\left(q_{2}-\sum_{j} \xi_{j}, t\right) \prod_{j=1}^{N} \frac{1}{\sqrt{2 \pi} \sigma_{j}} e^{-\frac{\xi_{j}^{2}}{2 \sigma_{j}^{2}}} d \xi_{j} .
$$

Notice that this is not a reduced density matrix in the ordinary sense, because the meaning of its off diagonal elements is not clear, mainly due to the fact that the variable $\xi_{j}(t)$ 's are not pure environment variables unless $\mathrm{t}=0$. However, the diagonal matrix elements $\rho(q, q ; t)$ have clear physical meaning: they are the probability density $\rho(q ; t)$. Hence we shall only address the diagonal elements in the following, even though the results for the off-diagonal elements are as simple as the diagonal elements.

It is easy to derive the following formula for an arbitrary function $f(\xi)$ :

$$
\underbrace{\iiint \ldots \int}_{N} f\left(\sum_{j} \xi_{j}\right) \prod_{j=1}^{N}\left(\frac{1}{\sqrt{2 \pi} \sigma_{j}} e^{-\frac{\xi_{j}^{2}}{2 \sigma_{j}^{2}}} d \xi_{j}\right)=\frac{1}{\sqrt{2 \pi} \sigma_{\xi}} \int f(\xi) e^{-\frac{\xi^{2}}{2 \sigma_{\xi}^{2}}} d \xi,
$$

with

$$
\sigma_{\xi}^{2}=\sum_{j=1}^{N} \sigma_{j}^{2}
$$

Therefore we have a very simple expression for the probability density:

$$
\rho(q, t)=\frac{1}{\sqrt{2 \pi} \sigma_{\xi}} \int|\psi(q-\xi, t)|^{2} e^{-\frac{\xi^{2}}{2 \sigma \xi^{2}}} d \xi .
$$


where $\sigma_{\xi}$ is the Brownian motion width, calculated by substituting eq.(10) into eq.( [13):

$$
\sigma_{\xi}^{2}(t)=\int_{0}^{\infty} \frac{\hbar}{2 m_{j} \omega_{j}}\left(\left|b_{j 1}(t)\right|^{2}+\omega_{j}^{2}\left|b_{j 2}(t)\right|^{2}\right) \operatorname{coth}\left(\frac{\hbar \omega_{j}}{2 k T}\right) \rho\left(\omega_{j}\right) d \omega_{j},
$$

and by replacing the sum over the bath oscillator number $\mathbf{j}$ with the spectral density $\rho\left(\omega_{j}\right)$ of the bath oscillators as given by eq.(6) of [1]:

$$
\rho\left(\omega_{j}\right)=\frac{2 \eta M}{\pi} \frac{m_{j} \omega_{j}^{2}}{c_{j}^{2}} .
$$

This width is zero initially, then approaches its final equilibrium value in a time interval of the order of $1 / \eta$. In low temperature limit, the equilibrium width is simplified to:

$$
\sigma_{\xi}^{2}(t=\infty)=\frac{\hbar}{2 \pi M \omega}\left[\frac{\pi}{2}+\arctan \left(\frac{\omega_{0}^{2}}{\eta \omega}\right)\right] .
$$

If $\eta \ll \omega_{0}$, this width happens to become the same as the width of the ground state of the system $\sigma_{0}^{2}=\hbar /\left(2 M \omega_{0}\right)$.

There are some subtleties to be addressed in future communications in the calculation of $\sigma_{\xi}(t)$ for finite t. They are associated with a logarithmic divergence of the integration over $\omega_{j}$ in eq.(15). The divergence is removed by introducing a cut-off frequency $\omega_{j}^{(\text {cut })}$ in eq.(15)). In the following, for simplicity, we consider only the low temperature limit. To get an idea of the behavior of $\sigma_{\xi}$, we plot $\sigma_{\xi}^{2} / \sigma_{0}^{2}$ in figure 1 , for $\omega_{j}^{(c u t)}=100 \omega$, and $\eta=0.1 \omega_{0}$. As a rough estimate, we have

$$
\sigma_{\xi}^{2} \approx \sigma_{0}^{2}\left(1-e^{-\eta t}\right)
$$

This function is not very sensitive to the specific value of $\omega_{j}^{(\text {cut })} / \omega_{0}$.

3. The wavefunction evolution. We assume that initially the wavefunction is a gaussian wave packet with minimum uncertainty and displaced from the center of the potential well by Z:

$$
\psi_{0}(q) \equiv \psi(q ; t=0)=\left(2 \pi \sigma_{0}^{2}\right)^{-1 / 4} e^{-\frac{(q-z)^{2}}{4 \sigma_{0}^{2}}} .
$$


Using the Green's function (derived as eq.(21) in [1] ):

$$
G\left(Q_{1}, Q_{0} ; t, 0\right)=\left(\frac{M \omega e^{\frac{1}{2} \eta t}}{2 \pi i \hbar \sin \omega t}\right)^{\frac{1}{2}} \exp \left[\frac{i M}{2 \hbar a_{2}}\left(a_{1} Q_{0}^{2}+\dot{a}_{2} e^{\eta t} Q_{1}^{2}-2 Q_{0} Q_{1}\right)\right] .
$$

we have:

$$
\begin{gathered}
\psi(q, t)=\int \psi\left(q_{0}, 0\right) G\left(q, q_{0} ; t, 0\right) d q_{0} \\
=\frac{\left(2 \pi \sigma_{0}^{2}\right)^{-1 / 4}}{\sqrt{a_{1}+i \omega_{0} a_{2}}} \exp \left\{-\frac{1}{4 \sigma_{0}^{2}\left(a_{1}+i \omega_{0} a_{2}\right)}\left[\left(\dot{a}_{2}+i \omega_{0} a_{2}\right) e^{\eta t} q^{2}-2 q z+a_{1} z^{2}\right]\right\},
\end{gathered}
$$

where $a_{1}$ and $a_{2}$ are the well known coefficients of the initial values $q_{0}$ and $\dot{q}_{0}$ for the solution of the classical damping equation $\ddot{q}+\eta \dot{q}+\omega_{0}^{2} q=0$ [1]. If $\eta \ll \omega_{0}$, we rewrite $a_{1}$ and $a_{2}$ (eq.(12) of [1]) approximately as:

$$
\begin{gathered}
a_{1}=e^{-\frac{\eta}{2} t}\left(\cos \omega t+\frac{\eta}{2 \omega} \sin \omega t\right) \approx e^{-\frac{\eta}{2} t} \cos \omega t \\
\omega_{0} a_{2}=e^{-\frac{\eta}{2} t} \frac{\omega_{0}}{\omega} \sin \omega t \approx e^{-\frac{\eta}{2} t} \sin \omega t, \quad \dot{a}_{2}(t) \approx a_{1}(t) .
\end{gathered}
$$

With this approximation, the wave function takes a simple and familiar form:

$$
\psi(q, t) \approx C(t) e^{-\frac{\left[q-q_{c}(t)\right]^{2}}{4 \sigma_{0}^{2} e^{-\eta t}}} e^{i k_{c}(t) q} .
$$

This is a gaussian wave packet centered at the classical damped orbit: $q_{c}(t)=e^{-\frac{\eta}{2} t} z \cos \omega t$, with momentum of

$$
\hbar k_{c}(t)=\hbar \frac{z}{2 \sigma_{0}^{2}} e^{\frac{\eta}{2} t} \sin \omega t \simeq M e^{\eta t}\left[\frac{d}{d t} q_{c}(t)\right] .
$$

This corresponds to the wave number of a particle of mass $M e^{\eta t}$. The coefficient $C(t)$ is simply a normalization constant (independent of q). Notice that even though the velocity of the classical motion $q_{c}(t)$ deceases as $e^{-\eta t / 2}$, the wavelength $2 \pi / k_{c}(t)$ still decays as $e^{-\eta t / 2}$ because the effective mass increases exponentially. Notice also that the width of the wave packet $\sigma_{0} e^{-\eta t / 2}$ deceases exponentially, so that the probability density distribution evolves into a $\delta$ function if Brownian motion can be neglected. Obviously, as time approaches infinity, 
the system approaches a classical limit. To provide a more clear picture of the evolution process, in Figure 2, we plot the real part of the wavefunction at 4 different times, assuming that the damping rate is $\eta=0.1 \omega_{0}$, and the initial displacement is $z=5 \sigma_{0}$.

4. The interference fringe wavelength. Now, we are ready to check if the wavelength decay is observable. If initially the wavefunction is a superposition of two such wave packets as eq.(19), one with $\mathrm{z}=0$, the other with $\mathrm{z} \neq 0$, the above results can be used to calculate straightforwardly the interference term (which has been studied in detail by CL using path integral technique[2,4]). The result will show how the Brownian motion erases the interference.

The initial wavefunction is:

$$
\psi_{0}(q)=\psi_{01}(q)+\psi_{02}(q)=C\left(e^{-\frac{q^{2}}{4 \sigma_{0}^{2}}}+e^{-\frac{(q-z)^{2}}{4 \sigma_{0}^{2}}}\right),
$$

where $C$ is a normalization constant.

Using eq.(27), eq.(22), and eq.(14), we find:

$$
\rho(q, t)=C^{2} \frac{\sigma_{0}}{\sigma_{\theta}}\left[e^{-\frac{q^{2}}{4 \sigma_{\theta}^{2}}}+e^{-\frac{\left(q-a_{1} z\right)^{2}}{4 \sigma_{\theta}^{2}}}+e^{-\frac{q^{2}+\left(q-a_{1} z\right)^{2}}{4 \sigma_{\theta}^{2}}} 2 \cos \left(\frac{q^{2}-\left(q-a_{1} z\right)^{2}}{4 \sigma_{\theta}^{2}} \frac{\omega_{0} a_{2}}{a_{1}}\right) e^{-\frac{z^{2}}{8 \sigma_{0}^{2}} \frac{\sigma_{\xi}^{2}}{\sigma_{\theta}^{2}}}\right]
$$

where

$$
\sigma_{\theta}^{2} \equiv \sigma_{0}^{2}\left(a_{1}^{2}+\omega_{0}^{2} a_{2}^{2}\right)+\sigma_{\xi}^{2}
$$

The last term in the parenthesis is the interference term, which agrees with the equation (2.9) of [4]. The notation is slightly different. For example, a part of the exponent of the last factor has been identified as the ratio of the Brownian motion width $\sigma_{\xi}^{2}$ over the full width $\sigma_{\theta}^{2}$ of the wave packet; $a_{1}$ is identified as the trajectory of a particle with initial position 1 but zero velocity, while $a_{2}$ corresponds to a particle with initial velocity 1 but position at the origin[1]. In every period, the maximum interference occurs when $a_{1}=0,(\omega t \approx(2 n+1) \pi / 2)$, as is indicated by the first factor of the interference term. In the following, we only discuss the time when $a_{1}(t)=0$. 
Since the wavelength decays exponentially in eq.(22), it is interesting to see whether the interference fringe wavelength also decays exponentially, and provides an observable phenomenon. We assume $\eta \ll \omega_{0}$, use the approximation eq.(24), and estimate the Brownian width by eq.(18). Then the growth of $\sigma_{\xi}^{2}$ and the decay of $a_{2}^{2}$ happen to cancel each other, and $\sigma_{\theta}^{2} \approx \sigma_{0}^{2}$ is nearly constant. The eq.(28) becomes:

$$
\rho(q, t) \approx 2 C^{2} e^{-\frac{q^{2}}{4 \sigma_{0}^{2}}}\left[1+\cos \left(k_{c 0} e^{-\frac{1}{2} \eta t} q\right) e^{-\frac{1}{2} k_{c 0}^{2} \sigma_{\xi}^{2}}\right]
$$

where $k_{c 0} \equiv \frac{z}{2 \sigma_{0}^{2}} \approx k_{c}\left(\omega t=\frac{\pi}{2}\right.$ ) (see eq.(26)). In order to have enough fringes the initial wavelength $2 \pi / k_{c 0}$ should be much smaller than the wave packet width $\sigma_{0}$. Hence the Brownian width increases to the initial wavelength in a time much shorter than the damping time $1 / \eta$ (the time for it to increase to $\sigma_{0}$ ). Therefore the eq.(30) shows that the fringe wavelength does not decay but increases slightly before the Brownian motion erases the interference pattern. Thus, in this well studied case, the exponential decay of wavelength is not experimentally observable!

5. How to observe the wavelength decay. If we carefully examine the calculation of the probability density eq.(14), we realize that the slight increase of fringe wavelength is due to the narrow wave packet width $\sigma_{0}^{2}$ and the convolution of the wavefunction with the Brownian motion in eq.(14). To see whether the wavelength decay is really observable, in the initial wavefunction eq.(27) we replace $\sigma_{0}$ by a different width $\sigma$. Let the ratio $\sigma_{0} / \sigma \equiv r$. Now, with a straightforward calculation as before, the probability density becomes:

$$
\rho(q, t)=C^{2} \frac{\sigma}{\sigma_{\theta}}\left[e^{-\frac{q^{2}}{4 \sigma_{\theta}^{2}}}+e^{-\frac{\left(q-a_{1} z\right)^{2}}{4 \sigma_{\theta}^{2}}}+e^{-\frac{q^{2}+\left(q-a_{1} z\right)^{2}}{4 \sigma_{\theta}^{2}}} 2 \cos \left(\frac{q^{2}-\left(q-a_{1} z\right)^{2}}{4 \sigma_{\theta}^{2}} \frac{\omega_{0} a_{2}}{a_{1}} r^{2}\right) e^{-\frac{z^{2}}{8 \sigma^{2}} \frac{\sigma_{\xi}^{2}}{\sigma_{\theta}^{2}}}\right],
$$

while

$$
\sigma_{\theta}^{2} \equiv \sigma^{2}\left(a_{1}^{2}+r^{4} \omega_{0}^{2} a_{2}^{2}\right)+\sigma_{\xi}^{2}
$$

Again, we use eq.(18) to calculate the fringe wavenumber (the coefficient of $q$ in the phase of 
the cosine function in eq.(31)). The fringe wavenumber is then found to grow exponentially until $t \simeq \ln \left(r^{2}-1\right) / \eta$, before it starts to decrease, due to the Brownian motion and the finite wave packet width. In the previously studied case, $r=1$, hence the wavelength decay is not observable. However, if $\mathrm{r}$ is sufficiently large, it becomes observable, as can be seen as follows. Now, for quite a long time, $\sigma_{\xi}^{2}$ can be neglected in eq.(32), while $\sigma_{\theta}^{2}$ decays exponentially, the interference pattern can then be simplified to (when $a_{1}=0$ and when the approximation eq.(24) is valid):

$$
\rho(q, t)=C^{2} \frac{2 \sigma}{\sigma_{\theta}} e^{-\frac{q^{2}}{4 \sigma_{\theta}^{2}}}\left[1+\cos \left(k_{c}(t) q\right) e^{-\frac{1}{2} k_{c}^{2}(t) \sigma_{\xi}^{2}}\right] .
$$

In the mean time, the visibility of the fringes also decays. The time when it is reduced

by a factor of e is determined by $\frac{k_{c}{ }^{2}(t) \sigma_{\xi}^{2}}{2}=1$, i.e., when the wavelength is about equal to the Brownian motion width. Using eq.(18), we find the time is $t \simeq \ln \left(1+8 \sigma_{0}^{2} / z^{2}\right) / \eta$. Thus large $\mathrm{r}$ and small $\mathrm{z}$ tend to give longer time to observe the wavelength decay before the fringes disappear. The number of visible fringes in the wave packet is $2 k_{c}(t) \sigma_{\theta} \simeq z / \sigma$ when $t \simeq(2 n+1) \pi / 2$, so $\mathrm{z}$ should be larger than $\sigma$ to have enough fringes. In figure 3 we plot the probability density at different times for a case with $z=3 \sigma_{0}$ and $\mathrm{r}=16$. A comparison of fig.3b and fig.3c clearly shows the wavelength decay and the visibility decay. In fig.3d, the coherence has been erased almost completely, long before the distribution width becomes $\sigma_{0}$.

\section{Acknowledgments}

The author thanks Prof. C.N. Yang for many sessions of stimulating discussions. The author also likes to thank C.P. Sun for interesting discussions. The work is performed under the auspices of the U.S. Department of Energy under Contract No. DE-AC02-76CH00016. 


\section{References}

1. L.H.Yu, C.P.Sun, Phys. Rev. A 49, 592 (1994)

2. A.O. Caldeira, A.J. Leggett, Ann. Phys. 149, 374 (1983), and Physica 121A, 587 (1983)

3. E. Kanai, Prog. Theor. Phys. 3, 440 (1948); S. Nakajima, Prog. Theor. Phys. 20, 948 (1958); R.Zwanzig, J. Chem. Phys. 33, 1338 (1960); I.R. Senitzky, Phys. Rev. 119, 670 (1960); G.W. Ford, M. Kac, P. Mazur, Jour. Math. Phys., 6, 504 (1965); P. Ullersma, Physica 32 (1966); M. D. Kostin, J. Chem. Phys. 57, 3589 (1972); K. Yasue, Ann. Phys. 114, 479 (1978); R.H. Koch, D.J. Van Harlingen, J. Clarke, Phys. Rev. Lett, 45, 2132 (1980); H. Dekker, Phys. Report, 80, 1 (1981); R. Benguria, M. Kac, Phys. Rev. Lett, 46, 1 (1981)

4. A.O. Caldeira, A.J. Leggett, Phys. Rev. A, 31, 1059 (1985)

5. R.P.Feynman, Statistical Mechanics, W.A.Benjamin, Inc. (1972) 


\section{Figure Captions}

Figure 1. The Brownian motion width as a function of time. The cut-off frequency $\omega_{j}^{(c u t)}=100 \omega, \eta=0.1 \omega_{0}$.

Figure 2. The time evolution of $\psi(q, t) . z=5 \sigma_{0}, \eta=0.1 \omega_{0}$.

Figure 3. The time evolution of the interference pattern. The probability density is in arbitrary unit. $z=5 \sigma_{0}, \eta=0.1 \omega_{0}, r=16, \omega_{j}^{(c u t)}=100 \omega$. 


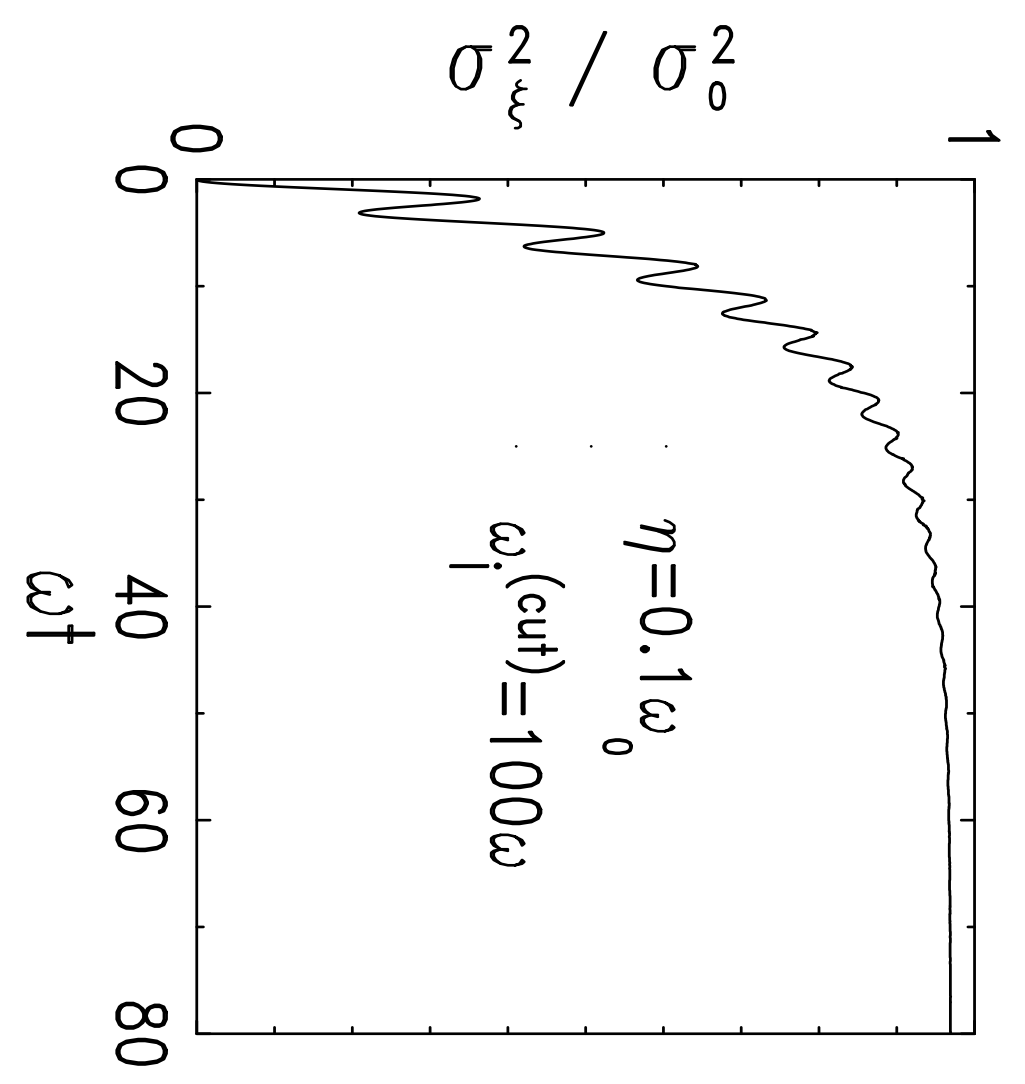




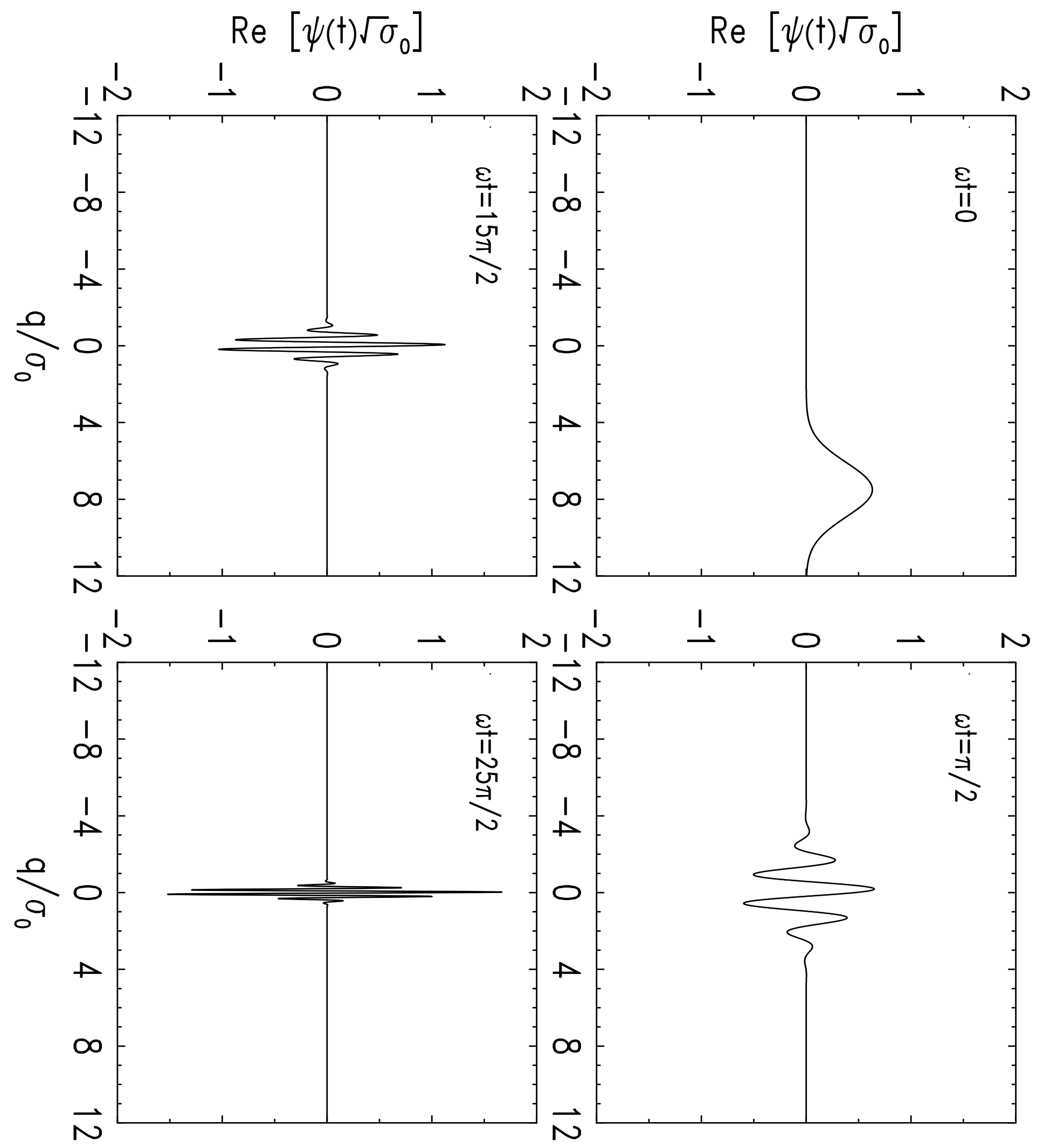




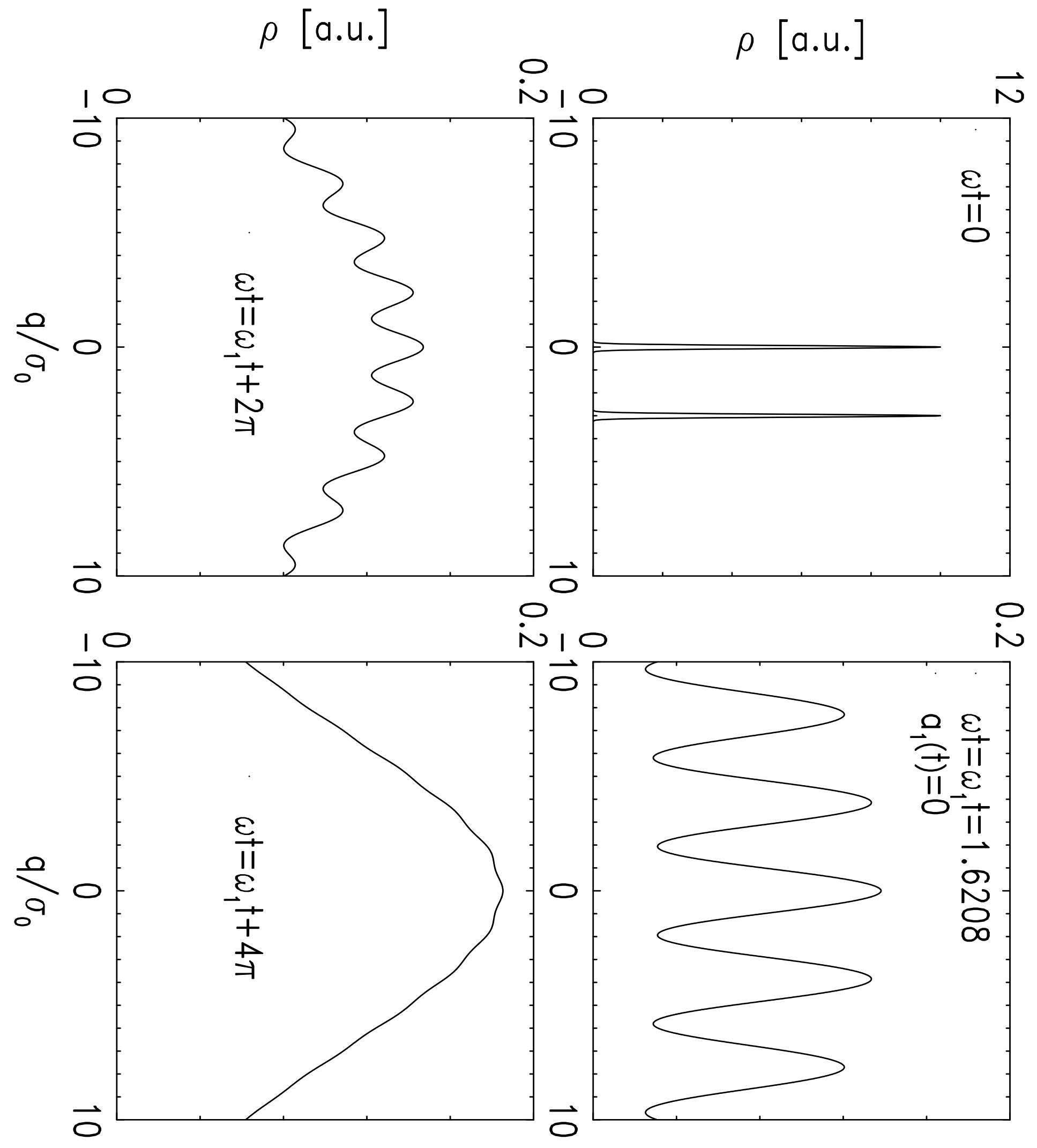

